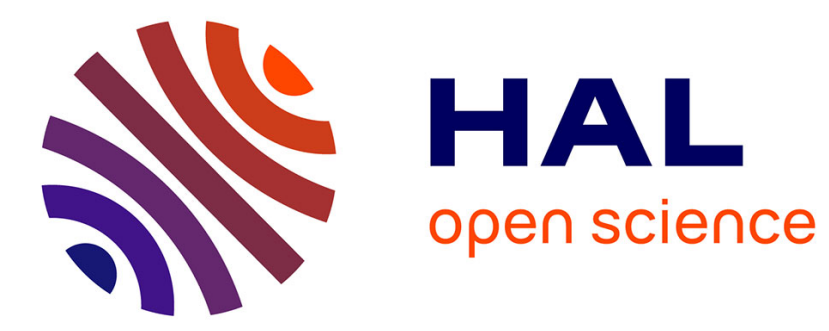

\title{
Improved Low Cost GPS Localization By Using Communicative Vehicles
}

Evangeline Pollard, Denis Gingras

\section{To cite this version:}

Evangeline Pollard, Denis Gingras. Improved Low Cost GPS Localization By Using Communicative Vehicles. 12th International Conference on Control, Automation, Robotics and Vision, ICARCV, Dec 2012, Guangzhou, China. hal-00735332

\section{HAL Id: hal-00735332 \\ https://hal.inria.fr/hal-00735332}

Submitted on 25 Sep 2012

HAL is a multi-disciplinary open access archive for the deposit and dissemination of scientific research documents, whether they are published or not. The documents may come from teaching and research institutions in France or abroad, or from public or private research centers.
L'archive ouverte pluridisciplinaire HAL, est destinée au dépôt et à la diffusion de documents scientifiques de niveau recherche, publiés ou non, émanant des établissements d'enseignement et de recherche français ou étrangers, des laboratoires publics ou privés. 


\title{
Improved Low Cost GPS Localization By Using Communicative Vehicles
}

\author{
Evangeline Pollard \\ INRIA \\ Domaine de Voluceau - Rocquencourt \\ B.P. 105 - 78153 Le Chesnay \\ evangelinepollard@gmail.com
}

\author{
Denis Gingras \\ Université de Sherbrooke \\ Faculty of Engeenering \\ EECE Department \\ Sherbrooke, Canada
}

\begin{abstract}
In this paper, a new collaborative localization method is proposed. On the assumption that the distance between two communicative vehicles can be calculated with a good precision, cooperative vehicle are considered as additional satellites into the user position calculation by using iterative methods. In order to limit divergence, some filtering process is proposed: Interacting Multiple Model (IMM) is used to guarantee a greater robustness in the user position estimation.
\end{abstract}

\section{INTRODUCTION}

Today, vehicles become more and more autonomous. Many Advanced Driver Assistance Systems (ADAS) are embedded in order to help the driver in the driving process. This is possible since vehicle are equipped with many sensors. Proprioceptive sensors (acceloremeter, gyrometer,...) provide information about the vehicle by itself such as its velocity or lateral acceleration. On the other hand, exteroceptive sensors, such as video camera, laser or GPS devices, provide information about the environment surrounding the vehicle or its localization. As data are noisy, inaccurate and can also be unreliable or unsynchronized, the use of data fusion techniques is required in order to provide the most accurate situation assessment as possible [1] as illustrated in Fig. 1. In other words, situation assessment consists in providing a local map, modeling the vehicle state by itself (position, velocity, acceleration, braking ability,...), but also potential obstacle states (position, velocity, type,...) like other vehicles, bicycles or pedestrians, and finally the environment state including weather conditions or road state.

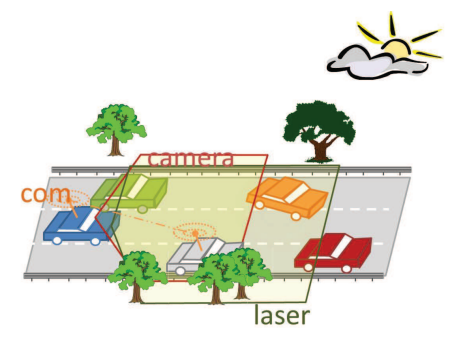

scene

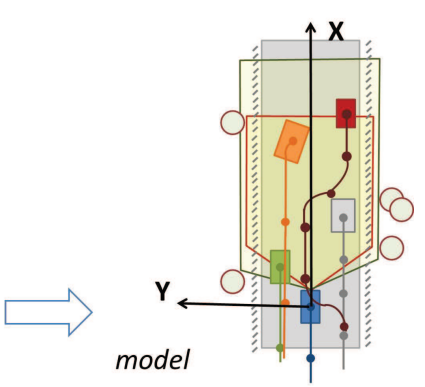

Figure 1. Perception task

Many ADAS are now commercialized such as Adaptive
Cruise Control (ACC), Lane change assistance systems or Collision avoidance systems. As a direct consequence of the electronics broadly used for vehicular applications, communication technologies are now being adopted as well. In order to limit injuries and to share safety information, research in driving assistance system is now orientating toward the cooperative domain. Vehicular Ad hoc Networks (VANETs) is thus considered as an essential development for future road safety and telematics applications. A dedicated bandwidth (Dedicated short-range communications: DSRC) for Intelligent Transportation Systems has been approved in US, Japan and Europe. It is exclusively used for Vehicle to Vehicle (V2V) and Vehicle to Road (V2R) communications.

Collaborative vehicular architectures is thus the main topic of several research projects around the world. The general idea is to combine the local perception of a set of individual vehicles into an extended map of their surrounding as illustrated in Fig. 2.

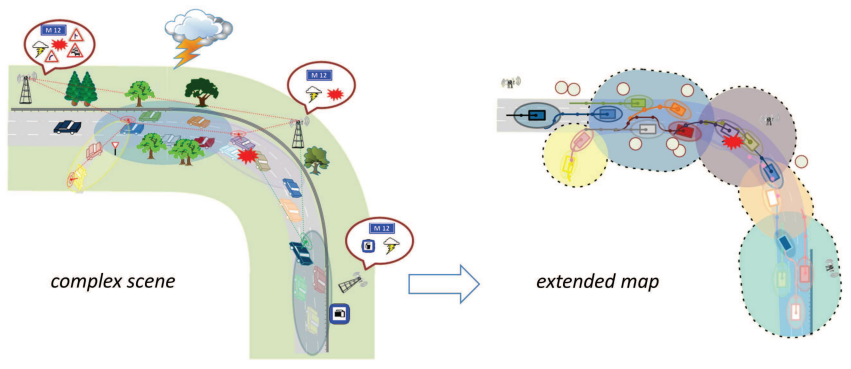

Figure 2. Extended Perception

Many applications of $\mathrm{V} 2 \mathrm{~V}$ or $\mathrm{V} 2 \mathrm{R}$ are now in progress such as Vehicle Collision Avoidance (VCA) or cooperative localization. If self-localization problem can be seen as already resolved since highly precise GPS (Global Positioning System) devices, like Differential GPS or RTK (Real Time Kinematic) exist, automotive constraints impose the use of very low cost sensors, whose reliability must be confirmed with complementary information coming from heterogeneous sensors. Moreover, ego-localization cannot be based only on 
one GPS sensor, whose signal can be temporary lost. That is why, the use of data fusion techniques is always required [2], [3].

In this paper, we focus on this last problem. On the assumption that the distance between two communicative vehicles can be calculated with a good precision, cooperative vehicle are considered as additional satellites into the user position calculation by using iterative methods. That was proposed in [4]. However, in order to limit divergence, some filtering process is proposed: Interacting Multiple Model (IMM) approach, widely presented and compared in the literature [5], guarantees a greater robustness to model unmatching, in order to provide the most accurate localization as possible. This highly improves localization precision. Moreover, more specifically in a urban context, it could guarantee the same level of precision even if satellites are occluded by urban objects.

The paper is organized as follows: in section II, GPS device positioning method is first presented. Then IMM approach, which is largely used in this paper is described in Sec. III. Finally, Sec. IV presents the collaborative method for GPS positioning method, while some simulation result demonstrates performance improvement of our method in Sec. V .

\section{GPS DEVICE POSITIONING}

\section{A. Positioning problem}

As shown in Fig. 3, user localization problem cannot be reduced to a trilateration problem. In fact, satellite-to-user distance is uncertain and if the number of satellites is higher than the number of unknown parameters, we want to provide the most accurate position estimation as possible, in other words, find the best compromise from the observations, as well as an estimation of the associated uncertainty.

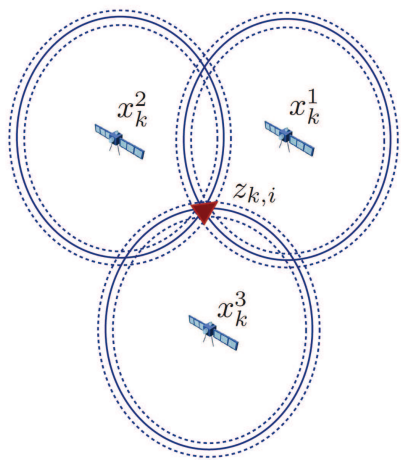

Figure 3. Trilateration with uncertainty. The red triangle represents the uncertainty area where the GPS device is positioned. Blue continue circles represents the pseudo-range of each satellite and the dashed circles their associated uncertainty

The pseudo-range $\rho_{k}^{i, j}$ between vehicle $i$ and satellite $j$ are computed by measuring the propagation time between the satellite and user receiver antenna. These pseudo-ranges are subject to errors due to several factors, such as the delay caused by the crossing of the atmosphere, the receiver noise and resolution offset, the multipath and shadowing effects, errors for the satellite clock or the ephemeris prediction.
Moreover, whereas satellites are equipped with very accurate atomic clock, this is not the case for GPS receivers for obvious cost reasons. Therefore, in addition to estimate the position $\left(x_{k, i}, y_{k, i}, z_{k, i}\right)$ of the GPS receiver at time $k$, the clock offset $\Delta t_{i}$ must be estimated.

This can be made by using the iterative method.

\section{B. Calculation of user position with iterative method}

In order to provide an estimation of the three dimension user position $\left(x_{k, i}, y_{k, i}, z_{k, i}\right)$ and the offset $\Delta t_{i}$, any satellite $j$ which has a line-of-sight with the GPS antenna $i$, sends the coordinate of their own position $\left(x_{k}^{(j)}, y_{k}^{(j)}, z_{k}^{(j)}\right)$. From this position, the distance between GPS antenna $i$ and satellite $j$ are linked by the following non-linear equation:

$$
\begin{aligned}
\rho_{k}^{i, j}= & \sqrt{\left(x_{k, i}-x_{k}^{(j)}\right)^{2}+\left(y_{k, i}-y_{k}^{(j)}\right)^{2}+\left(z_{k, i}-z_{k}^{(j)}\right)^{2}}+c \cdot \Delta t_{i} \\
& =g\left(x_{k, i}, y_{k, i}, z_{k, i}, t_{i}\right)
\end{aligned}
$$

The pseudo-range $\rho_{k}^{i, j}$ is calculated as the difference of Time of Arrival multiplied by the constant speed of light.

According to eq. 1, at least four satellites are required to solve the four-equation set. An algebraic solution of the GPS equations is proposed in [6]. However, this solution is not adapted to calculate the associated uncertainty and to take into account more than four satellites. In order to address this problem, an iterative method is used which is based on the linearization of eq. 1.

Starting with an approximate position $(\hat{x}, \hat{y}, \hat{z})$ and the time bias $\Delta \hat{t}_{i}$, an approximate pseudo-range $\hat{\rho}^{i, j}$ can be calculated:

$$
\hat{\rho}^{i, j}=\sqrt{\left(\hat{x}-x_{k}^{(j)}\right)^{2}+\left(\hat{y}-y_{k}^{(j)}\right)^{2}+\left(\hat{z}-z_{k}^{(j)}\right)^{2}}+c \cdot \Delta \hat{t}_{i}
$$

Using this new calculated pseudo-range and the Jacobian matrix $G$ of function $g$ described in eq. 1, the following equation can be written:

$$
\Delta \rho=\mathbf{G} \cdot \Delta \mathbf{x}
$$

with $\Delta \rho$ the vector containing the set of pseudo-range differences, $\Delta \mathrm{x}$ the vector of position difference as:

$$
\Delta \rho=\left[\begin{array}{c}
\hat{\rho}^{i, 1}-\rho_{k}^{1, j} \\
\vdots \\
\hat{\rho}^{i, n_{s}}-\rho_{k}^{1, n_{s}}
\end{array}\right], \Delta \mathbf{x}=\left[\begin{array}{c}
\hat{x}-\hat{x}_{k, i} \\
\hat{y}-\hat{y}_{k, i} \\
\hat{z}-\hat{z}_{k, i}
\end{array}\right]
$$

with $n_{s}$ the number of visible satellites.

And a new position can be incrementally calculated multiplying both sides of eq. 3 by $G^{T}$ if the matrix $G$ is not squared:

$$
\Delta \mathbf{x}=\left(\mathbf{G}^{\mathbf{T}} \cdot \mathbf{G}\right)^{-1} \mathbf{G}^{\mathbf{T}} \cdot \Delta \rho
$$

More information on the way to calculate $G$ can be found in [7]. This process is made until $\Delta \mathrm{x}$ becomes negligible. The final GPS measurement is denoted $\mathbf{z}_{k, i}$. 


\section{Calculation of the user position error}

The user position error depends on two factors. First, the relative geometry of visible satellites influences the error. The concept of dilution of precision (DOP) roughly interprets the geometric precision as ratio of position error to the range error. The smaller the ambiguity area, the better the precision (see Fig. 3). Second, the error for computing the pseudo-range distance between the GPS receiver and satellite position models the different error sources such as tropospheric/ionospheric delay, receiver noise and resolution offset, multipath and shadowing effects, errors for the satellite clock or the ephemeris prediction and is compiled into a User-Equivalent Range Error (UERE). The user position error is thus estimated as:

$$
(\text { GPSerror })=D O P \times(U E R E)
$$

Finally, associated measurement error matrix $\mathbf{R}_{k, i}$ is written as:

$$
\mathbf{R}_{k, i}=\left(G^{T} G\right)^{-1} \times \sigma_{U E R E}^{2}
$$

where $\sigma_{U E R E}$ is the pseudo range error factor.

\section{Interacting Multiple Model APPROACH DESCRIPTION}

\section{A. Estimation problem formulation}

Vehicle localization is done in a local three-dimensional frame. The state vector describing the vehicle state $\mathbf{x}_{k, i}$ is given by:

$$
\mathbf{x}_{k, i}=\left[\begin{array}{lllllllll}
\dot{x}_{k, i} & \ddot{x}_{k, i} & y_{k, i} & \dot{y}_{k, i} & \ddot{y}_{k, i} & z_{k, i} & \dot{z}_{k, i} & \ddot{z}_{k, i} & \omega_{k, i}
\end{array}\right]^{T}
$$

where $\omega_{k, i}$ is the orientation of the vehicle and $\left(x_{k, i}, y_{k, i}, z_{k, i}\right)$ corresponds to the position, $\left(\dot{x}_{k, i}, \dot{y}_{k, i}, \dot{z}_{k, i}\right)$ to the velocity and $\left(\ddot{x}_{k, i}, \ddot{y}_{k, i}, \ddot{z}_{k, i}\right)$ to the acceleration at time $k$ for the vehicle $i$ in the Cartesian model.

Assuming that the target motion is following the $r^{\text {th }}$ model $(\forall r \in[1, \ldots, m])$ represented by the $f^{(r)}$ function and the process noise $\mathbf{w}_{k}^{(r)}$, the vehicle state equation can be written as:

$$
\mathbf{x}_{k, i}=f^{(r)}\left(\mathbf{x}_{k-1, i}, \mathbf{w}_{k}^{(r)}\right)
$$

Measurement $\mathbf{z}_{k, i}$ describing the position of vehicle $i$ follows the measurement equation as following:

$$
\mathbf{z}_{k, i}=H \cdot \mathbf{x}_{k, i}+\mathbf{v}_{k, i}
$$

where $\mathbf{v}_{k, i}$ represents the measurement noise process at time $k$ defined as $\mathbf{R}_{k, i}=\mathbb{E}\left[\mathbf{v}_{k, i} \cdot \mathbf{v}_{k, i}^{T}\right]$ with the notation $\mathbb{E}[$. dedicated to the mathematical expectation.

By considering only, one motion model $M^{r}$, the state $\hat{\mathbf{x}}_{k \mid k, i}^{(r)}$ and covariance $\mathbf{P}_{k \mid k}^{(r)}$ estimates are calculated according to the Kalman filter as:

$$
\begin{gathered}
\hat{\mathbf{x}}_{k \mid k, i}^{(r)}=\mathbb{E}\left[\mathbf{x}_{k, i} \mid \mathbf{Z}^{k}, M^{r}\right] \\
\mathbf{P}_{k \mid k, i}^{(r)}=\mathbb{E}\left[\left(\mathbf{x}_{k, i}-\hat{\mathbf{x}}_{k \mid k, i}^{(r)}\right)\left(\mathbf{x}_{k, i}-\hat{\mathbf{x}}_{k \mid k, i}^{(r)}\right)^{T} \mid \mathbf{Z}^{k}, M^{r}\right]
\end{gathered}
$$

where $\mathbf{Z}^{k}$ refers to the cumulated measurements until time $k$.

In a multi-model context, the global estimation of the state $\hat{\mathbf{x}}_{k \mid k, i}$ and the covariance $\mathbf{P}_{k \mid k, i}$ of vehicle $i$ are calculated as a combination of the different estimates strongly of weakly weighted, according to the model occurrence probability $\mu_{k \mid k}^{(r)}$ at time $k$. Finally, the global state and covariance estimation are written as:

$$
\begin{gathered}
\hat{\mathbf{x}}_{k \mid k, i}=\sum_{r=1}^{m} \hat{\mathbf{x}}_{k \mid k, i}^{(r)} \cdot \mu_{k \mid k}^{(r)} \\
\mathbf{P}_{k \mid k, i}=\sum_{r=1}^{m}\left[\mathbf{P}_{k \mid k, i}^{(r)}+\left(\hat{\mathbf{x}}_{k \mid k, i}-\hat{\mathbf{x}}_{k \mid k, i}^{(r)}\right)\left(\hat{\mathbf{x}}_{k \mid k}-\hat{\mathbf{x}}_{k \mid k, i}^{(r)}\right)^{T}\right] \mu_{k \mid k}^{(r)}
\end{gathered}
$$

The main issue is now to calculate these model occurrence probabilities $\mu_{k \mid k}^{(r)}$.

\section{B. Model occurrence probability calculation}

The main assumption for using IMM is that jumps between the various system models are following a Markov chain process represented by the pre-defined transition matrix $\pi$. Then, model occurence probabilities can be calculated in four main steps:

- Mixing probabilities $\mu_{k}^{r \mid r^{\prime}}$ are first estimated at iteration $k, \forall\left(r, r^{\prime}\right) \in[1, \ldots, m]^{2}$ by using the transition matrix $\pi$.

- Each model estimate $\hat{\mathbf{x}}_{k \mid k, i}^{(r)}$ is mixed with the others model estimates and by using the mixing probabilities $\mu_{k}^{r \mid r^{\prime}}$ (interaction).

- By using the mixed model estimates, each model filter estimates the specific model state $\hat{\mathbf{x}}_{k \mid k, i}^{(r)}$ and covariance $\mathbf{P}_{k \mid k, i}^{(r)}$ in two steps: prediction and correction. This second step includes the computation of the measurement residual $\tilde{\mathbf{z}}_{k+1, i}^{(r)}$ and its covariance $\mathbf{S}_{k+1, i}^{(r)}$, called the model innovation.

- Assuming a Gaussian model, the model likelihood $\Lambda_{k+1}^{(r)}$ and probabilities $\mu_{k \mid k}^{(r)}$ can be computed as:

$$
\begin{gathered}
\Lambda_{k+1}^{(r)}=\frac{1}{\sqrt{\left|2 \pi \mathbf{S}_{k+1, i}^{(r)}\right|}} \exp \left(\frac{-\left(\tilde{\mathbf{z}}_{\mathrm{k}+1, \mathrm{i}}^{(\mathrm{r})}\right)^{\mathrm{T}}\left(\mathbf{S}_{\mathrm{k}+1, \mathrm{i}}^{(\mathrm{r})}\right)^{-1}\left(\tilde{\mathbf{z}}_{\mathrm{k}+1, \mathrm{i}}^{(\mathrm{r})}\right)}{2}\right) \\
\mu_{k+1 \mid k+1}^{(r)}=\frac{\mu_{k+1 \mid k}^{(r)} \Lambda_{k+1}^{(r)}}{\sum_{j} \mu_{k+1 \mid k}^{(j)} \Lambda_{k+1}^{(j)}}
\end{gathered}
$$

\section{IMM for ground maneuvering vehicles}

Various vehicle models are used to perform ego-localization with IMM. In order to derive these vehicle models, it is assumed that during a time period, the evolution sequence is shared into constant dynamic behaviors. The free motion evolution (no acceleration and no rotation) is represented by the Constant Velocity (CV) model. The longitudianl dynamics can be described as the Constant Acceleration (CA) model. For the lateral dynamics, a constant yaw rate model combined with a CV model is used, known as the Constant Turn model [8], [9]. 
The motion model jumps, as modeled as a Markovian transition process, is described by the transition matrix $\pi$. The estimation quality partially depends on the accuracy of this matrix. Values are obtained by using statistics on the vehicle motion, knowing that the frame of discernment for models is written $\Omega^{m}=\{C V, C A, C T\}$ :

$$
\pi=\left[\begin{array}{ccc}
0.9 & 0.08 & 0.02 \\
0.15 & 0.7 & 0.15 \\
0.04 & 0.16 & 0.8
\end{array}\right]
$$

The initial model probabilities $\mu_{0}$ following the same state vector as in eq. (17) is established as $\mu_{0}=\left[\begin{array}{lll}0.7 & 0.2 & 0.1\end{array}\right]$.

\section{COOPERATIVE GPS DEVICE POSITIONING}

The main proposed idea is really simple. If GPS positioning is made by trilateration considering satellite position and pseudo-range between satellites and ground vehicles, these same georeferenced vehicles can act as virtual satellites to improve the localization. As illustrated in Fig. 4, by using a communication system, the distance $d_{i i^{\prime}}$ between two vehicles $i$ and $i^{\prime}$ can be seen as a pseudo-range used in the equation set for user localization, as well as the communicative vehicle position.

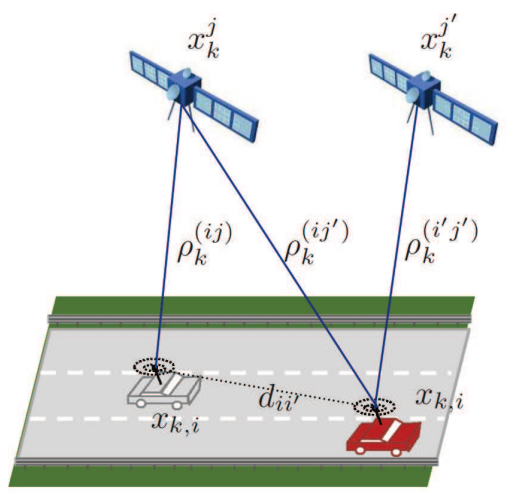

Figure 4. Two cooperative vehicles with two satellites

However, the crux of the issue is the way to calculate the distance between communicative vehicles. In [10], different methods to solve this problem of radio ranging in the DSRC context are presented such as Received Signal Strength (RSS), Time of Arrival (TOA), and Time Difference of Arrival (TDOA) for distance estimation between communicative vehicles. For example, Parker et al. [11] use RSS based intervehicle-distance measurements, vehicle kinematics, and road maps to estimate the relative positions of vehicles in a cluster. In [12], RSS and TOA are conjointly for indoor localization. However all these methods suffer of multipath and non line-of-sight (NLOS) problems. In [13], Le et al. suggests to conjointly use TDOA approach with Kalman filtering in order to robustify it. In [14], localization error is analyzed to correct multipath effects in a collaborative vehicular network. Hardware solutions are also an option. McCrady et al. [15] presented a two-way reciprocal ToA ranging technique that removes the need for any clock synchronization among receiver-transmitter pairs and provides high ranging accuracy $(\leq 1 \mathrm{~m})$ even in multipath scenarios.

In this paper, we supposed that the estimated distance $\hat{d}_{i i^{\prime}}$ between two vehicles is simply modeled as:

$$
\hat{d}_{i i^{\prime}}=d_{i i^{\prime}}+\nu
$$

where $\nu$ is a Gaussian noise process modeling uncertainty on the distance calculation.

In order to provide the most accurate estimation of the communicating vehicles and to limit divergence of the iterative method, their GPS measurement are filtered using a priori information on the target dynamics as shown in Fig. 5 in order to provide state estimated $\tilde{x}_{k, i}$. As ground vehicles are highly maneuverable, Kalman filtering is not adapted and an IMM is preferred as described in Sec. III.

Then, using the inter-distance module calculation, user position estimation can be improved by incorporating location of the surrounding communicative vehicles, in order to provide the final estimate $\hat{x}_{k, i}$ of the vehicle $i$ at time $k$.

\section{Simulation Result}

In the following, we present some simulation results that evaluate the performances of the proposed cooperative iterative method for GPS positioning. Performances are calculated with classical GPS positioning with iterative method, with cooperative GPS positioning with iterative method and also after filtering by using Kalman filter and IMM. Kalman $a$ priori model is a CV model with model noise $q_{K F}=1$ $\mathrm{m} / \mathrm{s}$. IMM models are CV, CA, CT models with model noises $q_{C V}=0.01, q_{C A}=2$ and $q_{C T}=0.2$.

Satellite position are computed with the satellites in Earth Centered, Earth Fixed (ECEF) coordinates position based on almanac data. GPS are first computed in ECEF coordinates and then converted into the local coordinates following chap. 2 of [7]. The pseudo range error factor $\sigma_{U E R E}$ is set up at $6.5 \mathrm{~m}$ as suggested in [7]. GPS scanning time is $1 \mathrm{~s}$. Limit elevation angle for satellites is $5 \hat{\mathrm{A}}^{\circ}$. Starting time is randomly generated and starting position is $(37.98,122.33,5)$ in ECEF coordinates. Noise on the distance calculation is $\nu=1 \mathrm{~m}$. Scenario time is limited to 50 iterations. Target trajectories are illustrated in Fig. 6. Three targets, following each other with a distance of $60 \mathrm{~m}$, are moving on the same road, with the same trajectory with an initial velocity of $30 \mathrm{~m} / \mathrm{s}$. They are descelerating before the curve (of $-1.41 \mathrm{~m} . \mathrm{s}^{-2}$ between iterations $k=26$ to $k=30$ ), turning (with $0.31 \hat{\mathrm{A}}^{\circ}$ between iterations $k=31$ to $k=35$ ) and accelerating (of $1,41 \mathrm{~m} . \mathrm{s}^{-2}$ between iterations $k=36$ to $k=40$ ).

The performances of positioning and tracking algorithms have been compared for the target in the middle, in Fig. 7, based on the Root Mean Square Error (RMSE) in horizontal position (top), $\mathrm{x}-\mathrm{y}$ position (middle) and 3D position (bottom) for 100 independent Monte Carlo runs. Average mean error are shown in Tab. I. From results presented in Fig. 6, it appears that cooperative approach highly improves GPS positioning (35\% in $3 \mathrm{D}$ and $50 \%$ in 2D). This improvement is constant over time. 


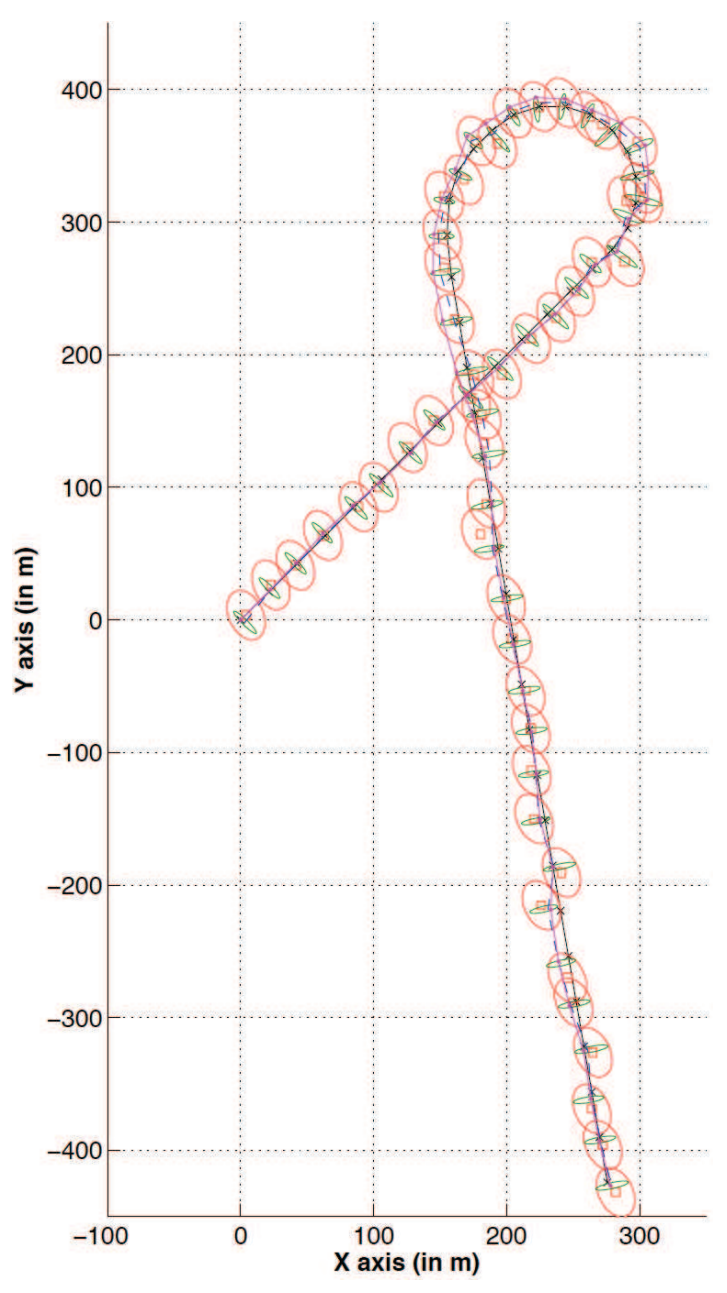

Figure 6. Target trajectory (in black), GPS measurement with uncertainty ellipses, (in red), cooperative GPS measurement with uncertainty ellipses (in green), Kalman estimates (in magenta) and IMM estimates (in blue).

\begin{tabular}{|c|c|c|c|c|}
\hline & IM & IM w. com & KF & IMM \\
\hline 2D RMSE & 7.77 & 3.93 & 3.19 & 2.96 \\
\hline 3D RMSE & 10.46 & 6.85 & 5.94 & 5.21 \\
\hline
\end{tabular}

Table I

AVERAGE ERROR IN 3D POSITIONING

Concerning filtering processes to take into account a priori model on the vehicle trajectory, Kalman filter and IMM yield to similar results when the target is moving according to the Kalman motion model (CV model). However, when the vehicle is maneuvering, Kalman filter yields to the filter divergence, while IMM positioning error stays stable. Numerical results shows an interesting improvement of performances (average error is less than $3 \mathrm{~m}$ ) proving that adapted filtering can highly help positioning task.

\section{CONCLUSION}

In this paper, we presented a new cooperative Iterative Method (IM) for GPS positioning. Based on the idea, than communicative vehicle can calculate their separating distance, communicative vehicle can act as virtual satellites and broadcast their own positioning. We proposed to improve the positioning of each vehicle before to introduce it into the cooperative iterative method. Simulation results prove that this approach can really improve self-positioning. This is a first work on the subject and now many perspectives can be considered. A first perspective would to integrate Inertial navigation system (INS) into the tracking process. Another perspective would to generalize the proposed method by using the GPSfree Positioning based on TOA distances proposed in [16] and to match relative positioning with dynamic positioning.

\section{ACKNOWLEDGEMENT}

This work is part of CooPerCom, a 3-year international research project (Canada-France). The authors would like to thank the National Science and Engineering Research Council (NSERC) of Canada and the Agence nationale de la recherche (ANR) in France for supporting the project.

\section{REFERENCES}

[1] D. Hall and J. Llinas, "An introduction to multisensor data fusion," Proceedings of the IEEE, vol. 85, no. 1, pp. 6-23, Jan 1997.

[2] —_, Handbook of Multisensor Data Fusion. CRC, 2001.

[3] R. Toledo-Moreo, M. Zamora-Izquierdo, B. Ubeda-Miarro, and A. Gómez-Skarmeta, "High-integrity IMM-EKF-based road vehicle navigation with low-cost GPS/SBAS/INS," IEEE Transactions on Intelligent Transportation Systems, vol. 8, no. 3, pp. 491-511, 2007.

[4] F. Berefelt, B. Boberg, J. Nygårds, P. Strömbäck, and S. Wirkander, "Collaborative gps/ins navigation in urban environment," in $\mathrm{ION} \mathrm{Na}$ tional Technical Meeting, 2004.

[5] S. Blackman and R. Popoli, Design and Analysis of Modern Tracking Systems. Artech House, 1999.

[6] S. Bancroft, "An algebraic solution of the GPS equations," IEEE Transactions on Aerospace and Electronic Systems, no. 1, pp. 56-59, 1985.

[7] E. Kaplan and C. Hegarty, Understanding GPS: principles and applications. Artech House Publishers, 2006.

[8] A. Ndjeng, S. Glaser, and D. Gruyer, "A multiple model localization system for outdoor vehicles," in Intelligent Vehicles Symposium. IEEE, 2007, pp. 1050-1055.

[9] R. Li and V. Jilkov, "Survey of maneuvering target tracking. Part V. Multiple-model methods," IEEE Transactions on Aerospace and Electronic Systems, vol. 41, no. 4, pp. 1255-1321, 2005.

[10] N. Alam, A. Balaei, and A. Dempster, "Range and range-rate measurements using DSRC: facts and challenges," in IGNSS Symposium, 2009.

[11] R. Parker and S. Valaee, "Vehicular node localization using receivedsignal-strength indicator," IEEE Transactions on Vehicular Technology, vol. 56, no. 6, pp. 3371-3380, 2007.

[12] M. Abusubaih, B. Rathke, and A. Wolisz, "A dual distance measurement scheme for indoor IEEE 802.11 wireless local area networks," in 9th IFIP International Conference on Mobile Wireless Communications Networks. IEEE, 2007, pp. 121-125.

[13] B. L. Le, K. Ahmed, and H. Tsuji, "Mobile location estimator with NLOS mitigation using Kalman filtering," in Wireless Communications and Networking, vol. 3, march 2003, pp. 1969-1973.

[14] N. Drawil and O. Basir, "Vehicular collaborative technique for location estimate correction," in IEEE 68th Vehicular Technology Conference. IEEE, 2008, pp. 1-5.

[15] D. McCrady, L. Doyle, H. Forstrom, T. Dempsey, and M. Martorana, "Mobile ranging using low-accuracy clocks," IEEE Transactions on Microwave Theory and Techniques, vol. 48, no. 6, pp. 951-958, 2000.

[16] S. Capkun, M. Hamdi, and J.-P. Hubaux, "GPS-free Positioning in Mobile Ad Hoc Networks," Cluster Computing, vol. 5, pp. 157-167, 2002. 
Satellite constellation

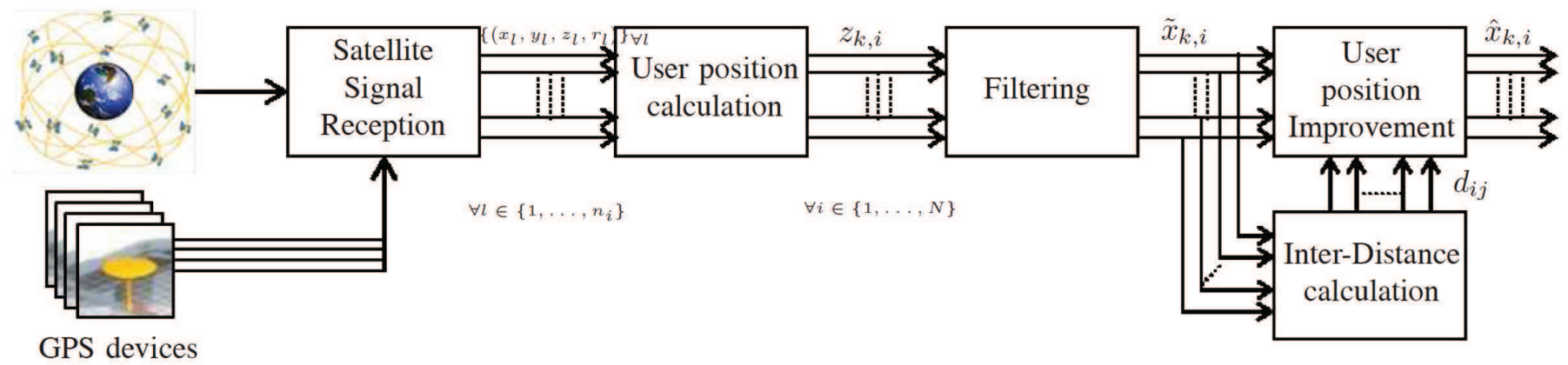

Figure 5. General scheme, with $n_{i}$ the number of visible satellites for vehicle $i$ and $N$ the number of vehicles
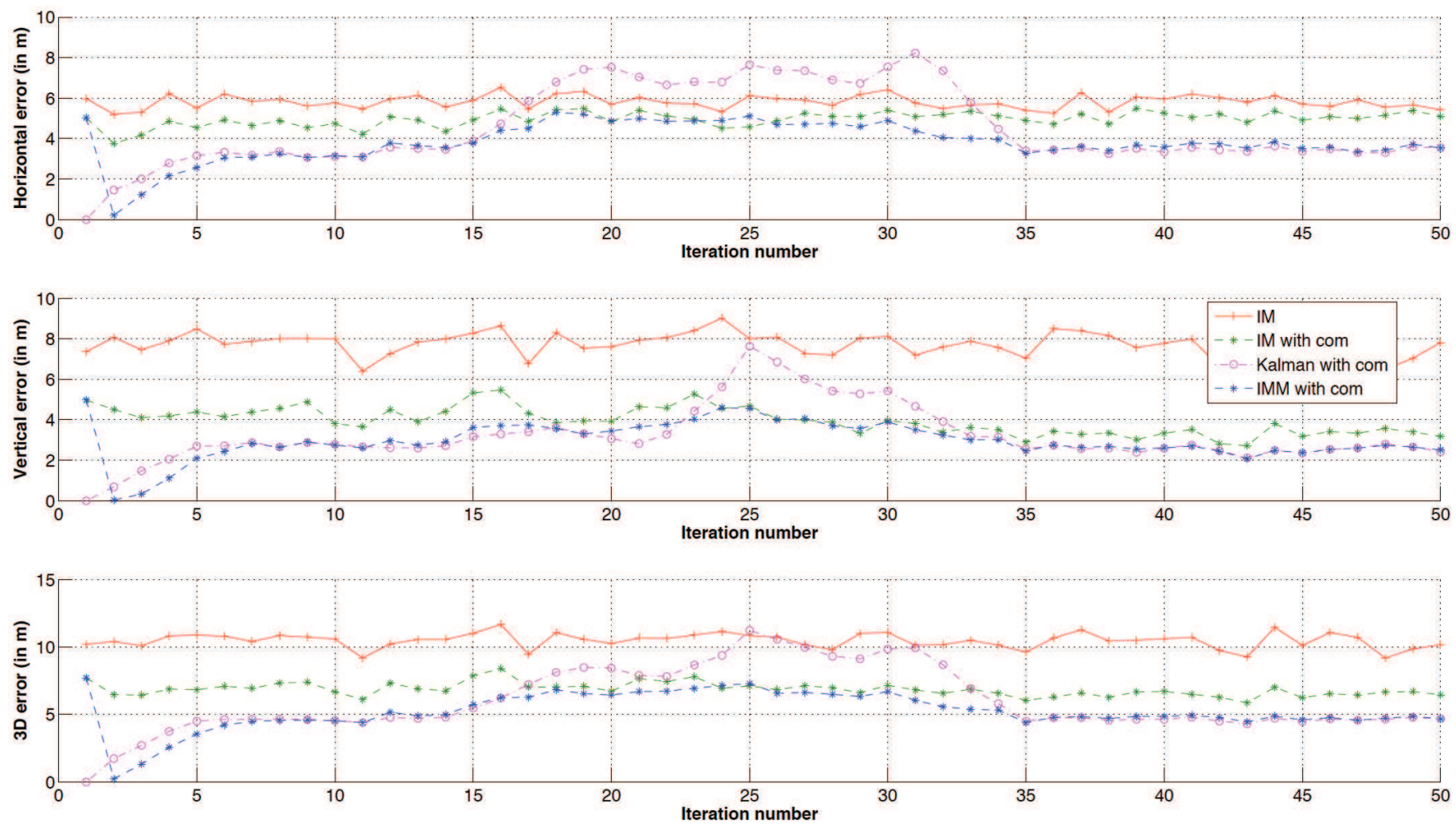

Figure 7. Positionning error 